\title{
Análisis Estructural del Fuselaje de un Sistema Aéreo No Tripulado de Monitoreo Volcánico
}

\section{Structural Analysis of the Airframe of an Unmanned Aerial Volcano Monitoring System}

\author{
DOI: $10.46932 / \mathrm{sfjdv2n5-054}$
}

Received in: Oct 1st, 2021

Accepted in: Dec 30th, 2021

\section{Miguel Angel Rosales Ochoa}

Maestro en Ingeniería Civil

Sección de Estudios de Posgrado e Invesitgación de ESIA Zacatenco del Instituto Politécnico Nacional

Calle Progreso \#531, Col. Americana

C.P.: 44160

Guadalajara, Jalisco

Santa María Tequepexpan,

San Pedro Tlaquepaque, Jalisco.

E-mail: rosalesmiguel.lan@ gmail.com

\section{José Félix Vázquez Flores}

Doctor en Ciencias con especialidad en Ingeniería Mecánica

ESIME U. P. Ticoman del Instituto Politécnico Nacional, México

Ticoman \#600, Col. San José Ticoman, Alcaldía Gustavo A. Madero, CDMX

C.P.: 07340

E-mail: bemol5808@gmail.com

\section{José Arturo Correa Arredondo}

Maestría en Ingeniería Aeronáutica

ESIME U. P. Ticoman del Instituto Politécnico Nacional, México

Ticoman \#600, Col. San José Ticoman, Alcaldía Gustavo A. Madero, CDMX

C.P.: 07340

E-mail: josearturocorrea@yahoo.com.mx

\section{RESÚMEN}

México es un país con más de 2,000 volcanes en su territorio, que de acuerdo con el Centro Nacional de Prevención de Desastres (CENAPRED), se encuentran en actividad 12 volcanes, por lo que realizar el monitoreo volcánico es de suma importancia, especialmente cuando los volcanes se encuentran cercanos a las poblaciones. La utilización de un UAS (sistema aéreo no tripulado) evita exponer a personas en ésta difícil tarea. En la ESIME Ticoman del Instituto Politécnico Nacional se trabaja en el proyecto "Tlapixki", un UAS para este fin, cuyo objetivo es asegurar que el fuselaje del avión soporte las cargas a las que será sometido, en este trabajo se presenta el análisis estructural del fuselaje en condición de despegue, con base en la reglamentación aérea y usando un paquete de elementos finitos. El material utilizado en la estructura es Aluminio 2024 T3, los resultados obtenidos del análisis muestran valores de esfuerzos mucho menores a los límites del material, mientras que, en el análisis modal, los desplazamientos generados son muy pequeños, indicando que la estructura del fuselaje es adecuada. 
Palabras claves: análisis estructural, método de los elementos finitos, fuselaje, modos de vibración, sistema aéreo no tripulado.

\begin{abstract}
Mexico is a country with more than 2,000 volcanoes in its territory, which according to the National Center for Disaster Prevention (CENAPRED), 12 volcanoes are active, so volcanic monitoring is essential, especially when volcanoes are close to populations. The use of a UAS (unmanned aerial system) avoids exposing people to this difficult task. The ESIME Ticoman of the National Polytechnic Institute is working on "Tlapixki" project, a UAS for this purpose, whose objective is to ensure that the aircraft's fuselage supports the loads to which it will be subjected, this work presents the structural analysis of the fuselage in take-off condition, based on aircrafts regulations and using a finite element software. The material used in the structure is Aluminum 2024 T3. The results obtained from the analysis show values of stress much lower than the limits of the material, while, in the modal analysis, the displacements generated are very small, indicating that the fuselage structure is adequate.
\end{abstract}

Keywords: structural analysis, finite element method, fuselage, shape modes, unmanned aircraft system.

\title{
1 INTRODUCCIÓN AL PROBLEMA
}

México es un país con más de 2,000 volcanes en su territorio, que de acuerdo con el Centro Nacional de Prevención de Desastres (CENAPRED) se encuentran en actividad 12 volcanes, Bárcena, Ceboruco, Citlaltépetl, Colima, El Chichón, Everman, Nevado de Toluca, Paricutín, San Martín, Tacaná, Tres Vírgenes y el Popocatépetl, siendo este último el de mayor interés por sus recientes y constantes emisiones de gases y ceniza. Ubicado en la zona centro del país, colindando con ciudades como Puebla, Ciudad de México, Estado de México, Morelos y Tlaxcala, siendo la más importante la CDMX en donde habitan 8.9 millones de personas (INEGI, 2015). Países como Japón, Estados Unidos, Costa Rica e Italia realizan el monitoreo, a través de un UAS (Sistemas Aéreos no Tripulados). En México no se cuenta con un UAS y se realiza a través de una caminata a la boca de los volcanes o a través de una avioneta en donde se expone al personal asignado.

\section{IMPORTANCIA DEL PROBLEMA}

Por lo anteriormente señalado, en la ESIME Ticoman del IPN (Instituto Politécnico Nacional), se desarrolla el proyecto “Tlapixki”, el cual consiste en el diseño y fabricación de un UAS (Almaraz Sánchez, 2017), con el fin de facilitar el monitoreo volcánico ya que el CENAPRED no cuenta con este recurso. El proyecto se enfoca en el Popocatépetl, debido a que es el segundo volcán más alto en la republica mexicana, con una altitud de 5,500m. La toma de fotografías, video, y muestras de gases permiten determinar el riesgo de una erupción. El trabajo que se describe en el presente, consiste en el análisis estructural del fuselaje del UAS, solamente en la condición de despegue, lo anterior se realizó de acuerdo al FAR 23 (Federal Aviation Regulations). 


\section{METODOLOGÍA}

La metodología utilizada en este proyecto consiste en un diseño base, configuración del fuselaje, un modelo mecánico preliminar, mallado del modelo, condiciones límites naturales y condiciones límites esenciales (Niu, 2001).

\section{DISEÑ BASE}

El diseño de la aeronave se realizó considerando varios aspectos, principalmente el que la cámara de fotografía y video deben tener una posición al frente en donde no se tengan objetos que interfieran, como por ejemplo la hélice u otros objetos, esto llevó a poner en la parte tracera el motor y la hélice, además de un botalón con el empenaje. Después, se consideró el equipo y la instrumentación que debería de llevar para el vuelo automatizado y por el otro lado, el de monitoreo volcánico (Almaraz Sánchez, 2017), lo cual conlleva a la configuración del fuselaje.

\section{CONFIGURACIÓN DEL FUSELAJE}

En base a lo anterior, se propuso una estructura semi-monocasco (figura 1). El fuselaje se encuentra dividido en nueve estaciones, cada estación cuenta con un sistema de dos cuadernas y doce largueros para la sección constante del fuselaje y ocho largueros para su sección variable, que van de cuaderna a cuaderna. Consta de una piel que envuelve a su esqueleto, esta piel tiene nueve laminas de aluminio con espesor de 1mm para cada estación, el material utilizado en el fuselaje es Aluminio 2024 T3.

Figura 1. Configuración del Fuselaje

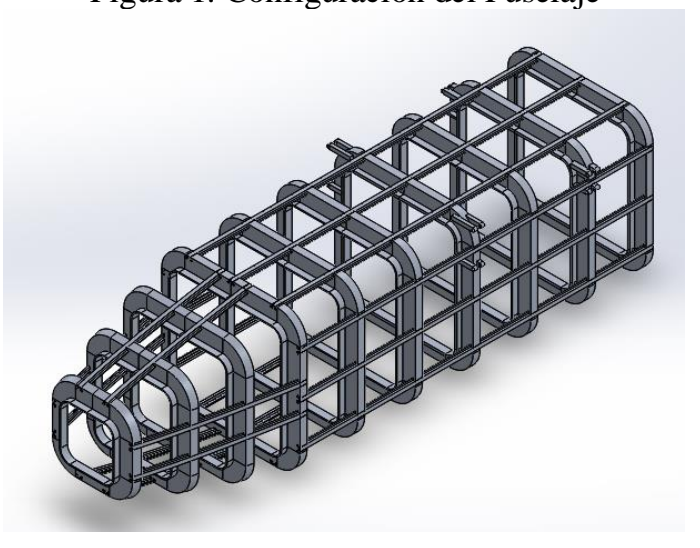

\section{MODELO MECÁNICO PRELIMINAR}

Se realizó el modelo de CAD del fuselaje en CATIA V5 (figura 2) y posteriormente se hizo el análisis en ANSYS. 
Figura 1. Modelo en CAD

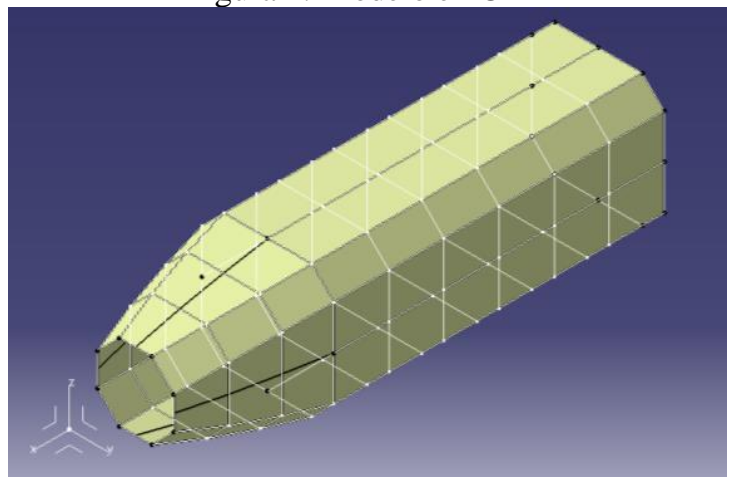

Una vez hecha la geometría del modelo en CATIA, se transportó al paquete ANSYS, para la asignación de las propiedades geométricas y posteriormente el mallado (figura 3).

Figura 3. Modelo en ANSYS

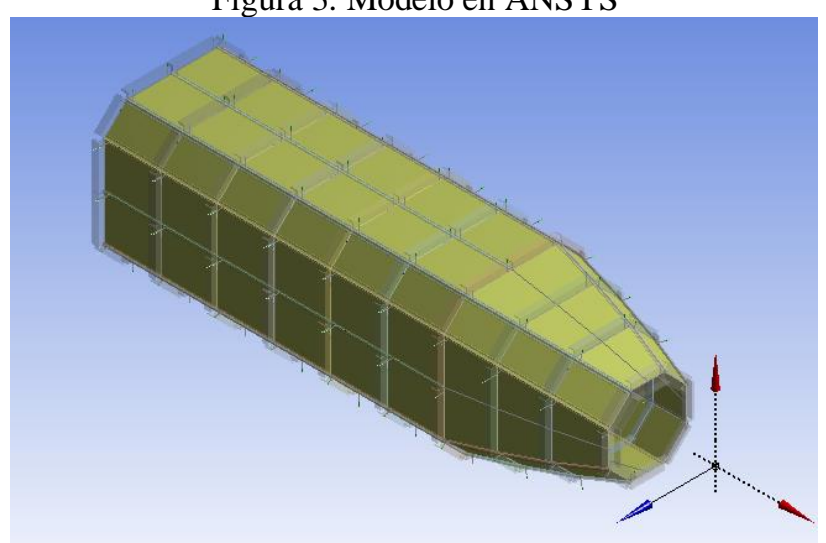

\section{MALLADO DEL MODELO}

Las cuadernas del fuselaje tienen una geometría cuadrada con las esquinas redondeadas con un perfil en "Z", mientras que, los largueros tienen un perfil tipo sombrero, ambos utilizan un elemento unidimensional. Por otra parte, la piel del fuselaje fue construida con elementos bidimensionales que pueden ser elementos triangulares o rectangulares. La geometría del fuselaje es una figura simétrica, lo que permite la utilización de elementos rectangulares en la piel y elementos lineales, cuidando la compatibilidad en los nodos (figura 4).

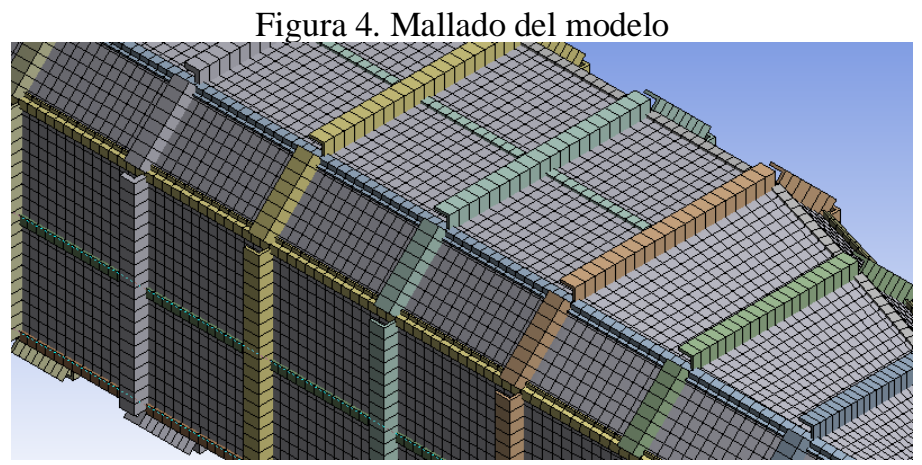




\section{CONDICIONES LÍMITE NATURALES}

Una de las condiciones más críticas de una aeronave es el despegue, el cálculo que se realizó fue en donde la aeronave está en el punto de rotación con una velocidad de $24.75 \mathrm{~m} / \mathrm{s}$, los motores están operando a máxima potencia y generan la tracción. En este punto la fricción que se produce por las ruedas es despreciable, ya que la sustentación es mayor al peso, otra de las fuerzas que se generan es el levantamiento y el peso máximo de despegue.

El peso máximo de despegue del UAS es de 40kg (Almaráz Sánchez, 2017) (tabla 1).

Tabla 1. Peso de los componentes del UAS

\begin{tabular}{|c|c|c|}
\hline Componente & $\begin{array}{c}\text { Masa } \\
\mathrm{kg}\end{array}$ & $\begin{array}{c}\text { Peso } \\
\mathrm{N}\end{array}$ \\
\hline Ala & 17.3 & 169.71 \\
\hline $\begin{array}{c}\text { Fuselaje, Tren de } \\
\text { aterrizaje y Equipo }\end{array}$ & 14.14 & 138.7 \\
\hline $\begin{array}{c}\text { Empenajes Horizontal, } \\
\text { Verticales y Botalones }\end{array}$ & 3.06 & 30.01 \\
\hline Motor & 5.5 & 53.95 \\
\hline Total & 40 & 392.4 \\
\hline
\end{tabular}

Para el cálculo de la fuerza de tracción se utilizó la velocidad de despegue o velocidad de $24.75 \mathrm{~m} / \mathrm{s}$.

$$
T=\frac{P_{T O}}{v_{R}}=\frac{15605 \mathrm{~W}}{24.75 \frac{\mathrm{m}}{\mathrm{s}}}=630.5 \mathrm{~N}
$$

El cálculo del levantamiento se hizo a través del método de red de vórtices (Almaraz Sánchez, 2017), utilizando una combinaciónb de un perfil aerodinámico NACA $65_{3} 618$ y un Eppler 604. Este perfil fue incluido en el programa XFOIL, el cual permite obtener los coeficientes de levantamiento y arrastre para distintos ángulos de ataque para un número de Reynolds del fluido.

Después de obtener los coeficientes del perfil aerodinámico, se realizó una corrección del coeficiente de levantamiento y arrastre del ala (Abbott \& Von Doenhoff, 1959) con las siguientes ecuaciones:

$$
\begin{gathered}
\mathrm{C}_{\mathrm{L}}=0.0759 \propto+0.3868 \\
\mathrm{C}_{\mathrm{D}}=0.0094+0.0566 \mathrm{C}_{\mathrm{L}}{ }^{2}
\end{gathered}
$$


La Tabla 2 muestra los coeficientes de levantamiento y arrastre del perfil aerodinámico y del ala, mientras que las curvas de los coeficientes de levantamiento contra ángulo de ataque se muestran en la gráfica de la figura 5 .

Tabla 2. Corrección de los Coeficientes de Levantamiento y Arrastre para distintos ángulos de ataque.

\begin{tabular}{|c|c|c|c|c|}
\hline$\alpha$ & $C_{l}$ & $C_{d}$ & $C_{L}$ & $C_{D}$ \\
\hline 9 & 0.9409 & 0.02272 & 1.07082693 & 0.07438793 \\
\hline 8 & 0.9572 & 0.01735 & 0.99482887 & 0.06549089 \\
\hline 7 & 0.9699 & 0.01336 & 0.91883081 & 0.05724852 \\
\hline 5 & 1.0181 & 0.00768 & 0.7668347 & 0.04272777 \\
\hline 4 & 0.9515 & 0.00713 & 0.69083664 & 0.0364494 \\
\hline 3 & 0.8519 & 0.00686 & 0.61483859 & 0.0308257 \\
\hline 2 & 0.7424 & 0.00684 & 0.53884053 & 0.02585666 \\
\hline 1 & 0.6272 & 0.00667 & 0.46284247 & 0.02154228 \\
\hline 0 & 0.5136 & 0.00639 & 0.38684442 & 0.01788257 \\
\hline-1 & 0.3984 & 0.0064 & 0.31084636 & 0.01487753 \\
\hline-2 & 0.2805 & 0.0062 & 0.23484831 & 0.01252716 \\
\hline-3 & 0.1615 & 0.0064 & 0.15885025 & 0.01083145 \\
\hline-4 & 0.0497 & 0.00905 & 0.08285219 & 0.00979041 \\
\hline-5 & -0.0578 & 0.00981 & 0.00685414 & 0.00940403 \\
\hline-6 & -0.1631 & 0.01057 & -0.06914392 & 0.00967232 \\
\hline-7 & -0.2657 & 0.01121 & -0.14514198 & 0.01059528 \\
\hline-8 & -0.3428 & 0.01214 & -0.22114003 & 0.0121729 \\
\hline-9 & -0.3605 & 0.01344 & -0.29713809 & 0.01440519 \\
\hline-10 & -0.3908 & 0.0153 & -0.37313615 & 0.01729214 \\
\hline-11 & -0.414 & 0.01819 & -0.4491342 & 0.02083376 \\
\hline-12 & -0.4442 & 0.02141 & -0.52513226 & 0.02503005 \\
\hline-13 & -0.453 & 0.02608 & -0.60113032 & 0.029881 \\
\hline-14 & -0.5732 & 0.02952 & -0.67712837 & 0.03538662 \\
\hline-15 & -0.6617 & 0.03326 & -0.75312643 & 0.04154691 \\
\hline-16 & -0.7592 & 0.03681 & -0.82912449 & 0.04836186 \\
\hline-17 & -0.2675 & 0.13708 & -0.90512254 & 0.05583148 \\
\hline
\end{tabular}


Figura 5. Comparación de las Curvas de $\boldsymbol{\alpha}$ contra Coeficiente de Levantamiento Coeficiente de Levantamiento vs. Ángulo de Ataque $(\alpha)$

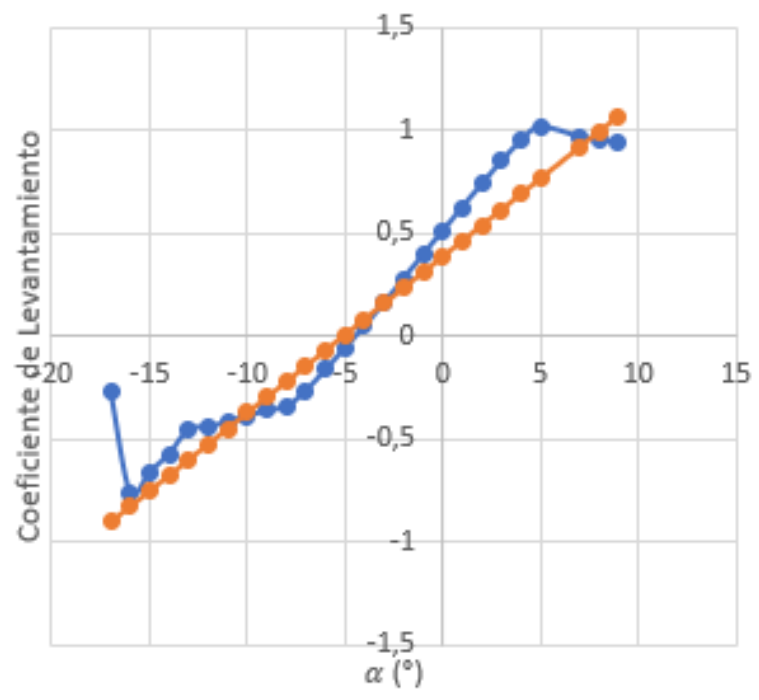

Posteriormente, se obtuvieron las fuerzas aerodinámicas en el despegue. La fuerza de sustentación se calculó considerando la superficie alar, mientras que, para la resistencia al avance se consideró la superficie de la piel del fuselaje que se encuentra en contacto con el viento.

$$
\begin{aligned}
& L=\frac{1}{2}\left(1.225 \frac{\mathrm{kg}}{\mathrm{m}^{3}}\right)\left(24.75 \frac{\mathrm{m}}{\mathrm{s}}\right)^{2}\left(1.65 \mathrm{~m}^{2}\right)(0.7668)=474.7 \mathrm{~N} \approx 475 \mathrm{~N} \\
& D=\frac{1}{2}\left(1.225 \frac{\mathrm{kg}}{\mathrm{m}^{3}}\right)\left(24.75 \frac{\mathrm{m}}{\mathrm{s}}\right)^{2}\left(1.11 \mathrm{~m}^{2}\right)(0.0427)=17.78 \mathrm{~N} \approx 18 \mathrm{~N}
\end{aligned}
$$

Se realizó la envolvente de vuelo del UAS Tlapixki de acuerdo con el FAR 23 (figura 6). La envolvente de vuelo está compuesta de dos diagramas que representan eventos independientes: las maniobras de la aeronave y las ráfagas para determinada altitud (Gudmundsson, 2014).

Figura 6. Envolvente de Vuelo del Tlapixki

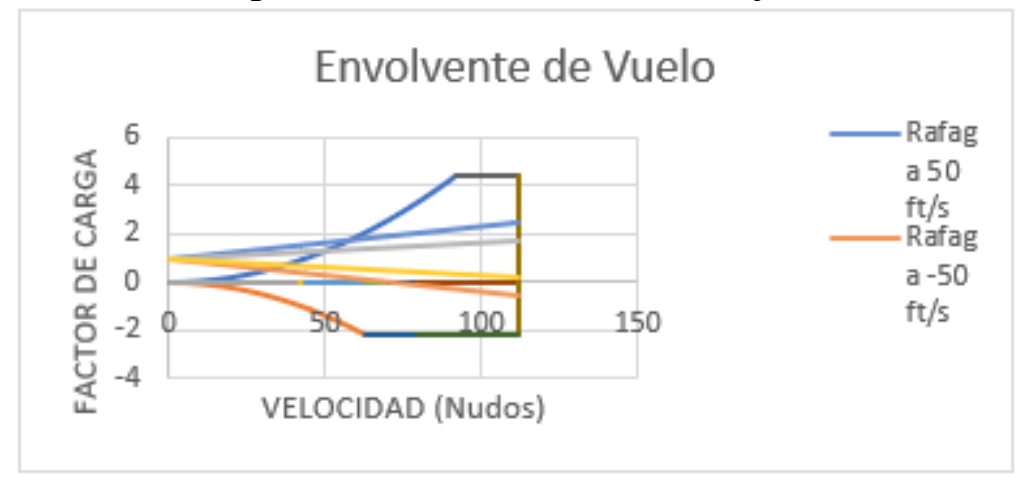


El FAR 23 propone revisar al fuselaje aplicando el factor de carga máximo de la envolvente de vuelo al peso y la sustentación de la aeronave (tabla 3), siendo un factor de 4.4.

Tabla 3. Factor de carga en el peso y sustentación.

\begin{tabular}{|c|c|c|}
\hline Tipo de Fuerza & $\begin{array}{c}\text { Magnitud } \\
N\end{array}$ & $\begin{array}{c}F * 4.4 \\
N\end{array}$ \\
\hline Peso del Ala & -169.71 & -746.72 \\
\hline $\begin{array}{c}\text { Peso del Fuselaje, Tren de } \\
\text { Aterrizaje y Equipo }\end{array}$ & -138.7 & -610.28 \\
\hline $\begin{array}{c}\text { Peso de Empenajes Horizontal, } \\
\text { Verticales y Botalones }\end{array}$ & -30.01 & -132.04 \\
\hline Peso del Motor & -53.95 & -237.38 \\
\hline Fuerza de Sustentación & 475 & 2090 \\
\hline
\end{tabular}

La sustentación, los pesos del ala, empenaje horizontal, empenajes verticales y botalones son fuerzas transmitidas al fuselaje en su unión con el ala, debido a su dirección estas se restan:

$$
F_{A-F}=L-W_{A}-W_{E H-E V-B}=2090 N-746.72 N-132.04 N=1211.24 N
$$

Donde $F_{A-F}$ es la fuerza en el herraje del fuselaje y donde se conecta el ala, la cual se transmite a las cuadernas (figura 7).

$$
F_{A-F}=\frac{1211.24 N}{0.6828 m}=1773.93 \frac{N}{m}
$$

Figura 7. Líneas de presión en la unión del ala y el fuselaje

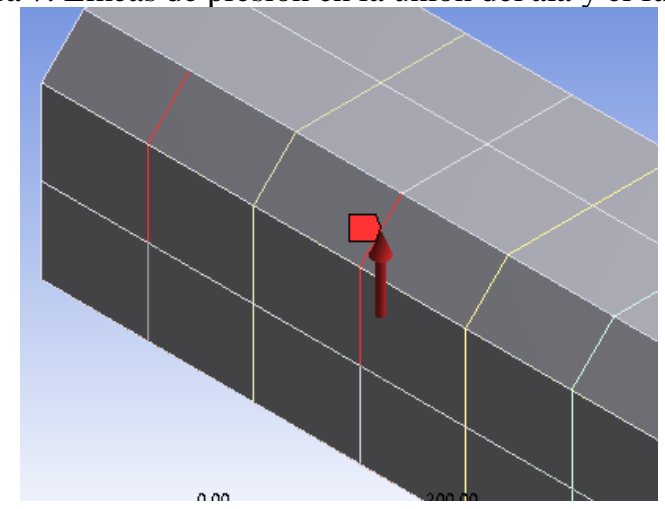

El peso del fuselaje, tren de aterrizaje y equipo se aplicó como una fuerza inercial que actúa en el peso propio del modelo. Esto se logró cambiando la densidad del material, relacionando la densidad y masa iniciales con la nueva masa, alcanzando la fuerza de $610.28 \mathrm{~N}$. 


$$
\rho_{1}=\frac{(62.21 \mathrm{~kg})\left(2780 \frac{\mathrm{kg}}{\mathrm{m}^{3}}\right)}{8.9283 \mathrm{~kg}}=19370.29 \frac{\mathrm{kg}}{\mathrm{m}^{3}}
$$

El motor del UAS se encuentra en la parte posterior del fuselaje, sujeto a la tapa-fuego. Esta placa está unida al fuselaje en la última cuaderna, por lo que el peso del motor fue distribuido como en su contorno y paralelo al eje de guiñada de la aeronave. Caso similar es el de la fuerza de tracción, que también fue distribuida alrededor de la cuaderna, pero con una ligera inclinación de $4^{\circ}$ alrededor del eje de cabeceo y $3^{\circ}$ alrededor del eje de guiñada (figura 8).

$$
\begin{gathered}
W_{M}=\frac{237.38 \mathrm{~N}}{1.0828 \mathrm{~m}}=219.22 \frac{\mathrm{N}}{\mathrm{m}} \\
T=\frac{630.5 \mathrm{~N}}{1.0828 \mathrm{~m}}=582.28 \frac{\mathrm{N}}{\mathrm{m}}
\end{gathered}
$$

Figura 8. Peso y Tracción del motor en el Fuselaje

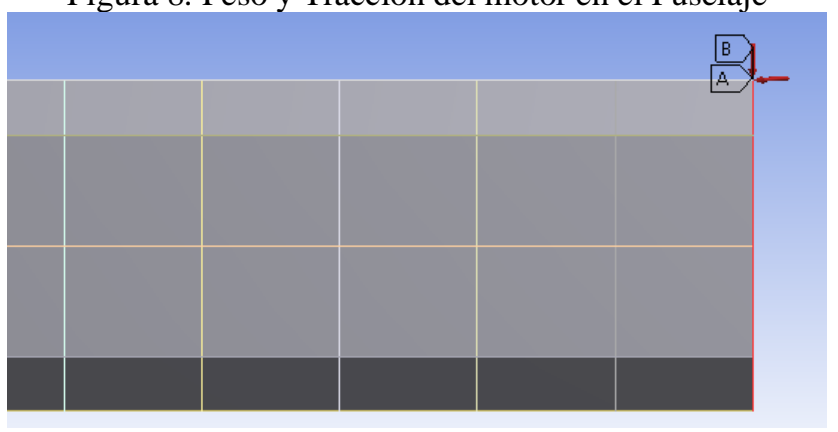

Finalmente, la resistencia al avance se distribuyó como una fuerza de presión tangencial y uniforme en cada panel de la piel (figura 9), considerando que el viento induce un flujo laminar en la superficie del modelo. La fuerza de presión en cada panel se obtuvo dividiendo la fuerza de arrastre entre la superficie del fuselaje. Con todas las condiciones límite naturales definidas para el despegue (figura 10), se prosiguió definiendo las condiciones límite escenciales del modelo.

$$
D=\frac{18 N}{1.11 m^{2}}=16.21 \frac{N}{m^{2}}
$$


Figura 9. Fuerza de arrastre distribuida en el fuselaje

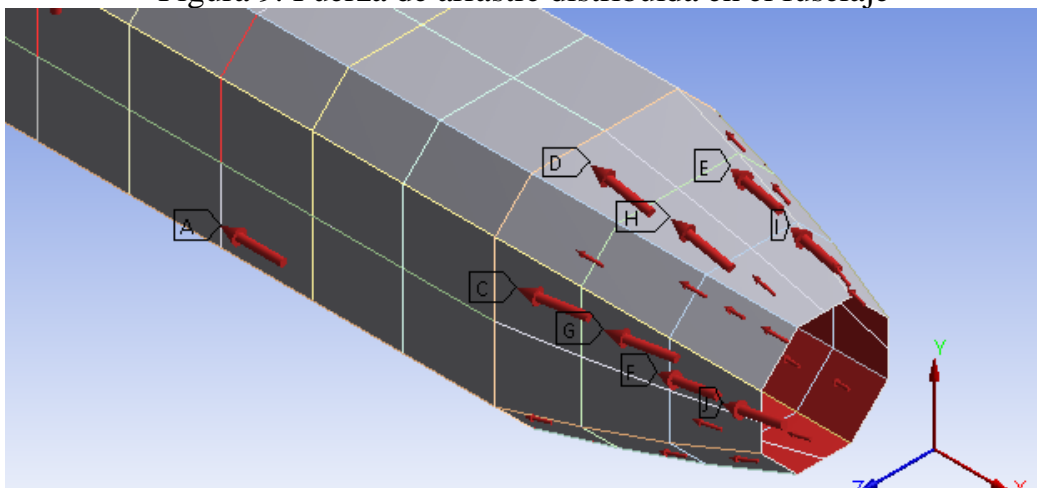

Figura 10. Condiciones Límite Esenciales en el Despegue

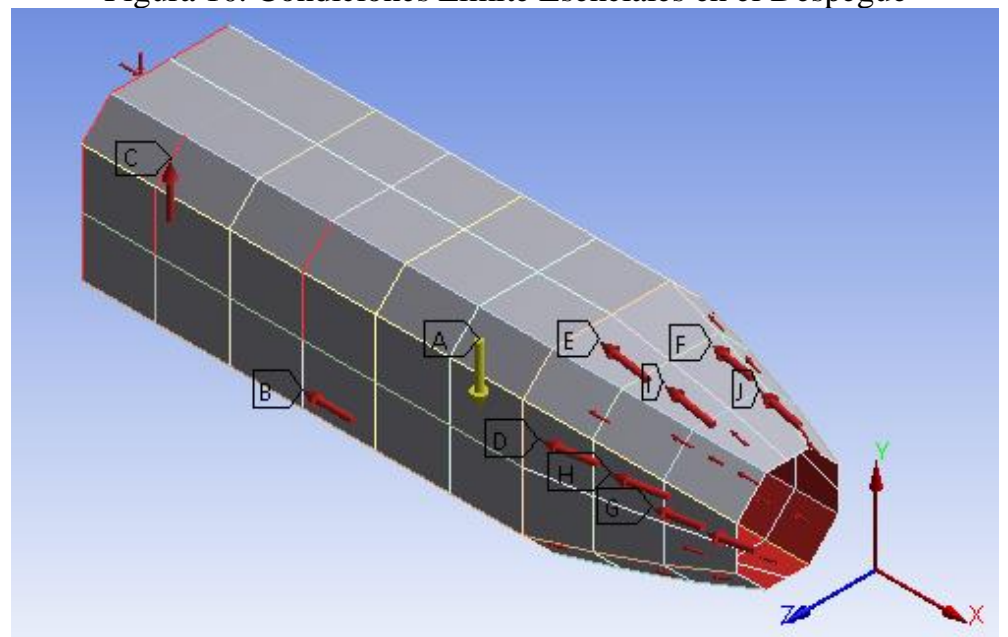

\section{CONDICIONES LÍMITE ESENCIALES}

Se utilizaron como apoyos los herrajes de sujeción del tren de nariz y tren principal de aterrizaje con el fuselaje para el despegue (figura 11). Mientras que para el análisis modal se estableció como condición de apoyo la unión de las alas y el fuselaje (figura 12).

Figura 11. Condiciones Límite Naturales en el despegue

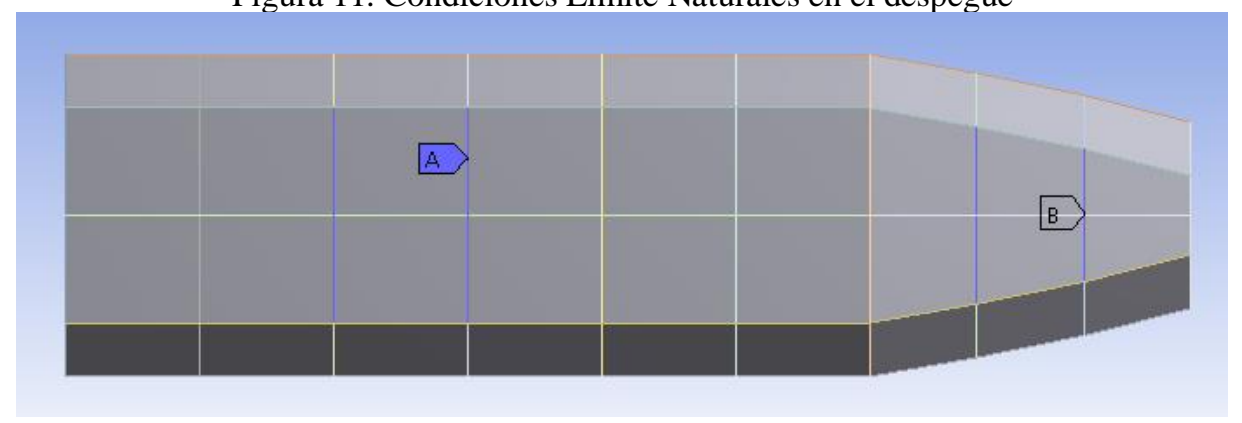


Figura 12. Condición de apoyo para el análisis modal

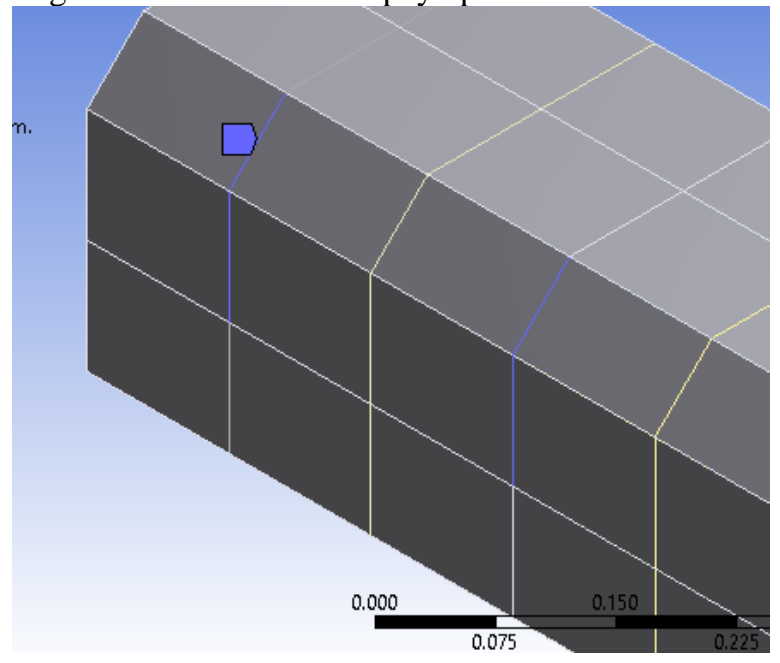

\section{RESULTADOS Y DISCUSIÓN}

\subsection{POST-PROCESO}

Después de realizar el análisis estructural en el despegue, se obtuvieron los estados de esfuerzos, deformaciones y desplazamientos del fuselaje. Se comparó el esfuerzo permisible con el esfuerzo máximo de Von Mises de 1.46MPa (figura 13), de la siguiente manera:

$$
\begin{gathered}
\sigma_{\text {Permisible }}=\frac{\sigma_{y}}{1.5}=\frac{2.8 \times 10^{8} \mathrm{~Pa}}{1.5}=186 \times 10^{6} \mathrm{~Pa} \\
\frac{\sigma_{\text {Permisible }}}{\sigma_{\text {máx }}}=\frac{186 \times 10^{6} \mathrm{~Pa}}{1.42 \times 10^{6} \mathrm{~Pa}}=130.9
\end{gathered}
$$

Con esto se comprueba que la estructura es capaz de soportar las cargas en el despegue, teniendo un desplazamiento máximo de $0.01 \mathrm{~mm}$ como se observa en la figura 14. Además, al utilizar el factor de carga de 4.4 enunciado en el FAR 23, se prueba que el fuselaje cae dentro de la envolvente de vuelo, siendo una estructura segura en esta condición. 
Figura 13. Esfuerzos de Von Mises en el fuselaje

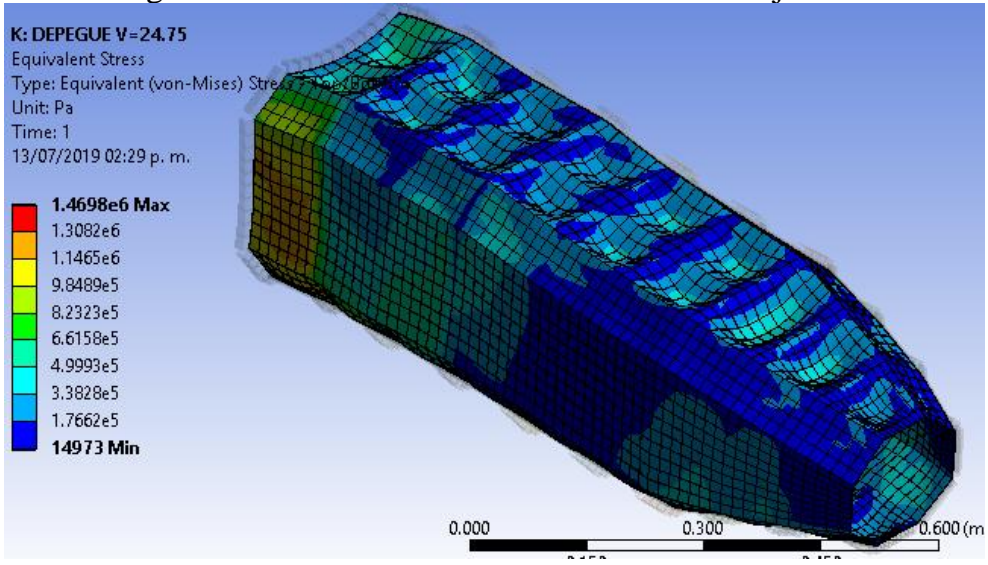

Figura 14. Desplazamientos en el fuselaje

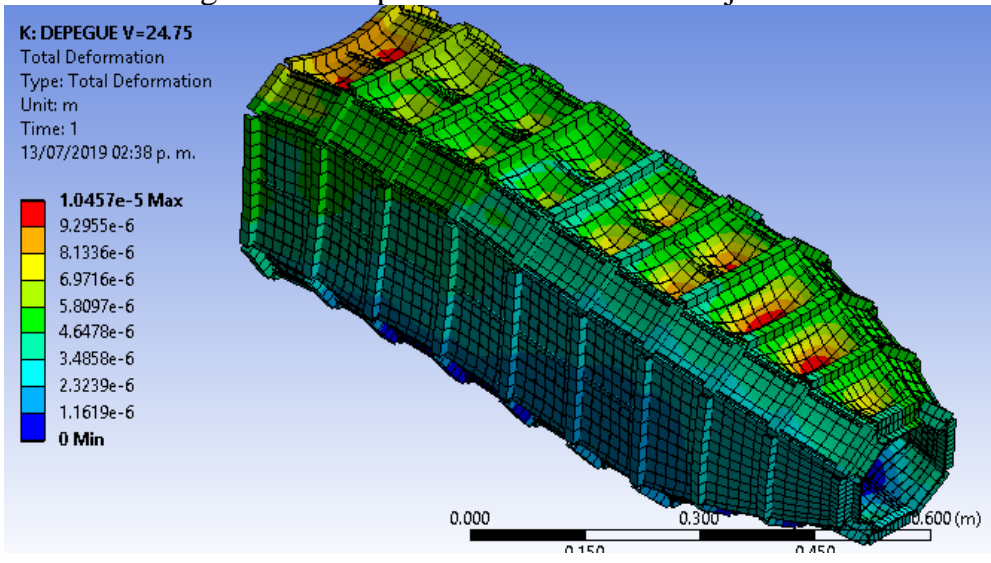

\subsection{MODOS DE VIBRACIÓN}

Se solicitaron 3 formas de vibrar para el anáñisis y se muetran a continuación:

Figura 15. Modo 1, flexión alrededor del eje z

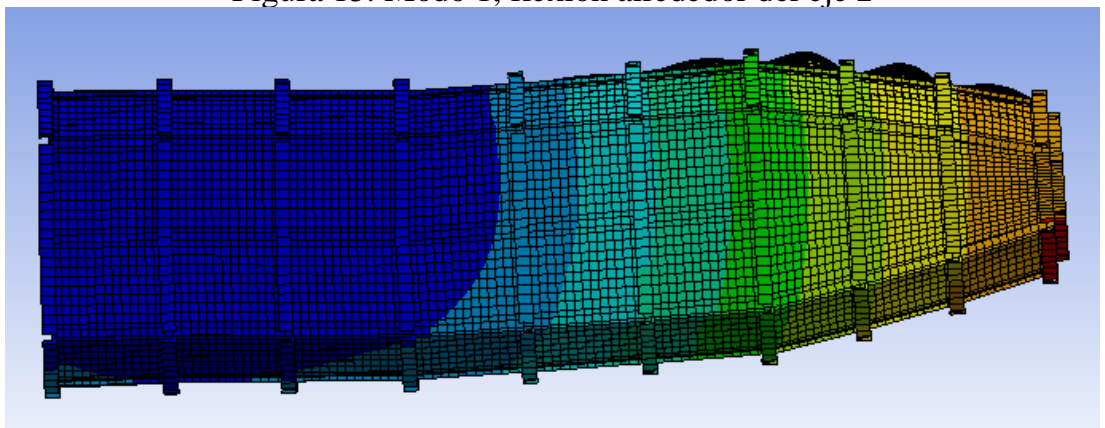


Figura 16. Modo 2, flexión alrededor del eje y

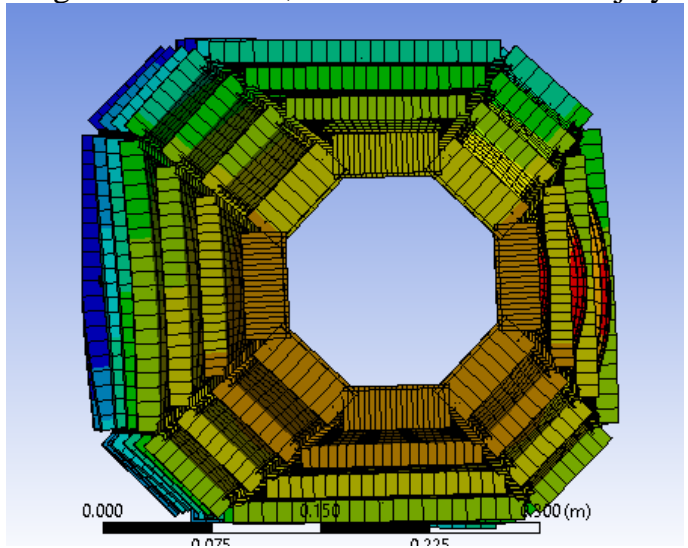

Figura 17. Modo 3, Torsión alrededor del eje $\mathrm{x}$

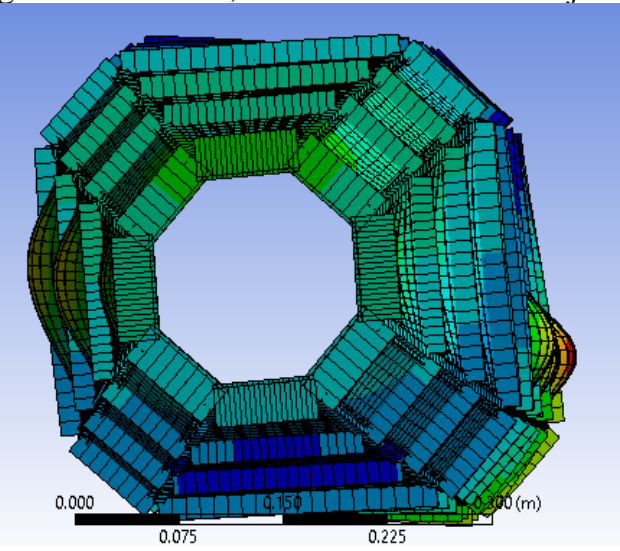

Finalmente se muestra una tabla con las frecuencias y periodos naturales de la estructura:

Tabla 4. Frecuencias y Perdiodos Naturales de lo modos de vibración

\begin{tabular}{|c|c|c|c|}
\hline Modo & $\begin{array}{c}\text { Frecuancia } \\
(\mathrm{Hz})\end{array}$ & $\begin{array}{c}\text { Periodo } \\
(\mathrm{s})\end{array}$ & $\begin{array}{c}\text { Desplazamiento } \\
\text { máximo }(\mathrm{mm})\end{array}$ \\
\hline 1 & 298.59 & 0.003349 & 15.3 \\
\hline 2 & 310.45 & 0.003221 & 18.8 \\
\hline 3 & 506.38 & 0.001974 & 29.9 \\
\hline
\end{tabular}

\section{CONCLUSIÓN}

Del análisis estático realizado al fuselaje en la condición de despegue, se encontró que, bajo la condición de carga, la estructura es capaz de resistir o soportar las cargas a las que será sometido, sin alcanzar el esfuerzo de cedencia del material (aluminio 2024 T3) aún considerando el factor de seguridad que define la reglamentación aérea a través del FAR 23, el cual es de 1.5. 
El primer modo de vibración (figura 15), resulta una flexión con respecto al eje Z o transversal, sobre el que se produce el cabeceo. El segundo modo de vibración también presenta una flexión (figura 16), pero esta es alrededor del eje $\mathrm{Y}$ o de guiñada y finalmente el tercero (figura 17), se obtiene una deformación angular, alrededor del eje X o de alabeo. Estos valores llevan a deformaciones muy pequeñas, pero si se recomienda realizar un análisis experimental para validar el cálculo.

Nota.- ver figura 10 para posición de los ejes. 


\section{REFERENCIAS}

Abbott, I. H., \& Von Doenhoff, A. E. (1959). Theory of Wing Sections. Toronto: Dover Publications.

Almaraz Sánchez, E. C. (2017). Diseño Aerodinamico de un Sistema Aereo no tripulado (UAS) para vigilancia Volcanica en México (Tesis de Grado ed.). Ciudad de México, México: SEPI ESIME Zacatenco.

Gudmundsson, S. (2014). General Aviation Aircraft Design: Applied Methods and Procedures. Waltham Massachusetts: ELSEVIER.

INEGI. (2015). Cuéntame... $\quad$ de $\quad$ Oéxico. http://cuentame.inegi.org.mx/monografias/informacion/df/poblacion/

Niu, M. (2001). Airframe Stress Analysis and Sizing (Segunda Edición ed.). Hong Kong: Conmilit Press LTD. Recuperado en Marzo de 2019 